\title{
Early Macrophage Activation in Preterm Newborns and Respiratory Disease
}

\author{
Anna Milan ${ }^{1}$ Elena Priante ${ }^{1}$ Daniel Nardo ${ }^{1}$ Francesca Tosato ${ }^{2}$ Giorgia Pantano ${ }^{2}$ Eugenio Baraldi ${ }^{1}$ \\ Patrizia Zaramella ${ }^{1}$ \\ ${ }^{1}$ Department of Women's and Children's Health, Neonatal Intensive \\ Care Unit, Padova University Hospital, Padova, Italy \\ 2 Department of Laboratory Medicine, Padova University Hospital, \\ Padova, Italy \\ Address for correspondence Patrizia Zaramella, MD, Neonatal \\ Intensive Care Unit, Department of Women's and Children's Health, \\ Padova University Hospital, Via Giustiniani 3, Padova 35128, Italy \\ (e-mail: patriz.zaramella@gmail.com).
}

J Child Sci 2017;7:e110-e119.

\begin{abstract}
Monocyte-macrophages have a role in host defense and tissue remodeling. Classically activated (M1) and alternatively activated (M2) macrophages from preterm newborns are analyzed, and the role in acute respiratory distress syndrome (RDS) and bronchopulmonary dysplasia (BPD) is evaluated. Observational study was conducted on the blood samples (BSs) and tracheal aspirates (TAs) collected at 48 to 72 hours of life in preterm newborns. Flow-cytometry was performed to identify monocytes and M1 or M2. Prenatal factors, gestational age, birth weight, acute RDS and BPD were assessed and related to the M1 and $M 2$ levels and M2/M1. One hundred nine subjects were included, and 100 were followed up. $\mathrm{M} 1$ and $\mathrm{M} 2$ increase and decrease, respectively, according to the gestational age and birth weight. Higher $\mathrm{M} 2$ and lower $\mathrm{M} 1$ levels in TAs were found after maternal chorioamnionitis.

Keywords

- bronchopulmonary dysplasia

- macrophage

- preterm newborn

- respiratory distress syndrome BPD patients have low M1 with high M2 in blood samples (BSs), as well as in tracheal aspirates (TAs). No relation was found between activation pattern and prenatal variables or the RDS grade. The correlation between gestational age or birth weight and M1 could reflect a more mature macrophage system, capable to push undifferentiated macrophages toward the classical pathway. We speculate that adequate early classical macrophage activation could be crucial to protect lungs from post-natal injuries, preventing the development of BPD.
\end{abstract}

\section{Introduction}

Bronchopulmonary dysplasia (BPD) is the respiratory chronic lung disease of preterm newborns with a reported incidence of $23 \%$ in infants born at 28 weeks or less gestation and increasing up to $73 \%$ in infants born at 23 weeks. ${ }^{1}$ At the histopathological level, BPD is the result of an arrest in lung development with disruption of distal lung growth and impaired alveologenesis and angiogenesis.

It is reported that when bone marrow-derived angiogenic progenitor cells (BMDAC) have been injected in an animal model of BPD, they showed a complete restoration of lung structure. ${ }^{2}$ A more detailed characterization revealed that they expressed myeloid/macrophage markers, and when cultured, they differentiated into macrophages supporting the hypothesis that myeloid/macrophage lineage could take part in lung restoration. In view of their plasticity and ability to react to different environmental signals, macrophages have been classified according to their activation pathway into two main groups: ${ }^{3,4}$ classically activated or alternatively activated cells. Classically activated macrophages (M1) are activated during cell-mediated immune response by IFN- $\gamma$ and tumor necrosis factor (TNF) produced by natural killer (NK) cells, antigen received

May 9, 2017

accepted after revision

July 10, 2017
DOI https://doi.org/

10.1055/s-0037-1605579.

ISSN 2474-5871.
Copyright @ 2017 Georg Thieme

Verlag KG Stuttgart · New York
License terms

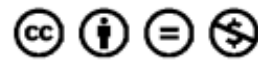


presenting cells, and T-cells. These macrophages have microbicidal activity; they can produce nitric oxide, $\mathrm{O}_{2}$ radicals, and inflammatory cytokines (e.g., Interleukin [IL]-1, IL-6, and IL-23). Their importance in inflammatory reaction and their pivotal role in host defense to infections and injury have been extensively studied. ${ }^{5}$ Alternatively activated macrophages (M2) are mainly activated by IL-4 and IL-13 released during tissue injury. Under the control of these mediators, M2 produce high levels of anti-inflammatory cytokines (IL-1 ra, IL-10, and transforming growth factor beta [TGF- $\beta$ ]) and Arginase- 1 that converts arginine to ornithine, precursor of polyamines, and collagen necessary for matrix remodeling. M2 do not produce nitric oxide or $\mathrm{O}_{2}$-radicals, and they lack in direct killing ability. Finally they promote angiogenesis, ${ }^{6}$ tissue repairing, and homeostasis. ${ }^{7}$

While the importance of the monocyte compartment in tissue repair mechanism has already been demonstrated in other organs, ${ }^{8}$ the specific role of macrophages in the physiological and pathological lung development have not been clarified yet. It is well known that M1 activation is associated with production of reactive oxygen species and inflammatory cytokines, which can substantially impair vascular function. Indeed, impaired microcirculatory function in newborns with respiratory distress syndrome has been directly documented. ${ }^{9}$ In newborns, microcirculation, endothelial dysfunction, and vascular injury are huge and impacting fields of investigation in correlation with macrophages polarization.

This study aims to analyze the early balance between two major macrophage subpopulations (M1 and M2) and their relationship with prenatal factors (chorioamnionitis, cesarean section [CS], twins pregnancy, and antenatal steroids administration), gestational age (GA), and birth weight (BW). A further aim was to assess whether the levels of macrophages subtypes at birth are associated with the severity of acute disease (RDS, fraction of inspired oxygen- $\mathrm{FiO}_{2}$ ) and chronic lung disease (mechanical ventilation [MV] time, $\mathrm{O}_{2}$ therapy duration, and BPD) in a preterm newborns group.

\section{Materials and Methods}

\section{Study Design}

Preterm newborns admitted to a level III Neonatal Intensive Care Unit of the Women's and Children's Health Department, Padova, Italy from January 2013 to April 2015 were included in this observational longitudinal study. Exclusion criteria were major malformations and major cardiovascular defects. An ethylenediaminetetraacetic acid (EDTA) blood sample (BS $=0.5 \mathrm{~mL}$ ) during routine patient evaluation was collected at 48 to 72 hours of life, median age 50 hours, was stored in culture medium (Roswell Park Memorial Institute medium [RPMI]), and was immediately sent to the local department of laboratory medicine. Overall patients being intubated at the same time, a TAs sample was also collected during a routine endotracheal (ET)-tube suction. Both samples were analyzed by flow cytometry to identify M1 and M2. Clinical data assessment of the enrolled patients includes (1) prenatal factors: histological chorioamnionitis (presence of more than 10 polymorph nuclear leukocytes/high-powered field, ${ }^{10}$
CS, twins pregnancy, and antenatal steroids administration); (2) biological features: GA and BW; (3) severity of acute RDS at sampling: radiological RDS grade ${ }^{11}$ and $\mathrm{FiO}_{2}$ levels; and (4) presence and severity of chronic lung disease: mechanical ventilation (MV) time, $\mathrm{O}_{2}$ therapy duration, and BPD (oxygen support at 36 weeks postmenstrual age). ${ }^{12}$ Our Neonatal Intensive Care Unit (NICU) policy is that the infant breathing spontaneously, with RDS, is first treated with continuous positive airway pressure (CPAP or high-flow nasal cannula) or neonatal nasal intermittent positive pressure ventilation (NIPPV). Patients with neonatal RDS require endotracheal intubation and administration of surfactant. Moreover intubation and MV are indicated for patients who fail to respond to less invasive forms of ventilation and are carried out with low tidal volumes (4-6 mL/kg) with permissive hypercapnia (partial pressure of carbon dioxide $\left[\mathrm{PaCO}_{2}\right] 50-55 \mathrm{~mm} \mathrm{Hg}$ ). In our practice, intubation and MV are initiated when one or more of the following criteria are verified: respiratory acidosis, i.e., an arterial $\mathrm{pH}<7.2$ and partial pressure of arterial carbon dioxide $\left(\mathrm{PaCO}_{2}\right)>60$ to $65 \mathrm{~mm} \mathrm{Hg}$; hypoxemia documented by an arterial partial pressure of oxygen $\left(\mathrm{PaO}_{2}\right)<50 \mathrm{~mm} \mathrm{Hg}$, despite oxygen supplementation, or when the fraction of inspired concentration $\left(\mathrm{FiO}_{2}\right)$ exceeds 0.40 on CPAP; and apnea. Overall our TAs were obtained from newborns intubated and were submitted to MV (synchronized or high-frequency oscillatory) before surfactant administration. Since sparse data are suitable in the field of macrophage phenotype and BPD ${ }^{13}$ or RDS, an ex ante sample size calculation seems difficult to reach. We choose to collect TAs and BSs within the third day of life to avoid confounding biased infections or the inflammatory factors rising in patients submitted to prolonged ventilation.

\section{Sample Analysis}

Sample preparation was performed according to the National Committee for Clinical Laboratory Standards (NCCLS) (H42A2) guidelines. ${ }^{14}$ One hundred microliters of EDTA BSs or TAs, preserved in a specific culture medium (RPMI), were added to an aliquot of monoclonal antibody as suggested by the manufacturer and had been incubated for 15 minutes at room temperature. The following color combination of monoclonal antibodies was used: CD14-PE, CD45-ECD, CD33-PeCy5, and CD16-PeCy7 (Beckman-Coulter). Staining was always undertaken on the day of sampling.

After incubation, BSs erythrocytes were lysed with TQ-prep (Beckman-Coulter), while TAs samples erythrocytes had been lysed with a lysing solution with ammonium chloride for 5 minutes. Samples were then acquired on the flow cytometer.

Four-color analysis was performed on a Navios flow cytometer (Beckman-Coulter). Acquisition was run until 30,000 and 10,000 events were detected, for BSs and TAs respectively. Data analyses were made with CXP software (Beckman-Coulter). Using the appropriate "gating" approach, the relative frequencies were determined for total monocytes/macrophages (CD33 $+/$ SS $^{\text {low }}$ ) and, among these, for classically activated macrophages (CD14++/CD16-) and alternatively activated macrophages (CD14+/CD16+). ${ }^{8,13,15}$ M1 and M2 levels were expressed as percentage of macrophage subtypes among the whole population - Fig. 1 A. 

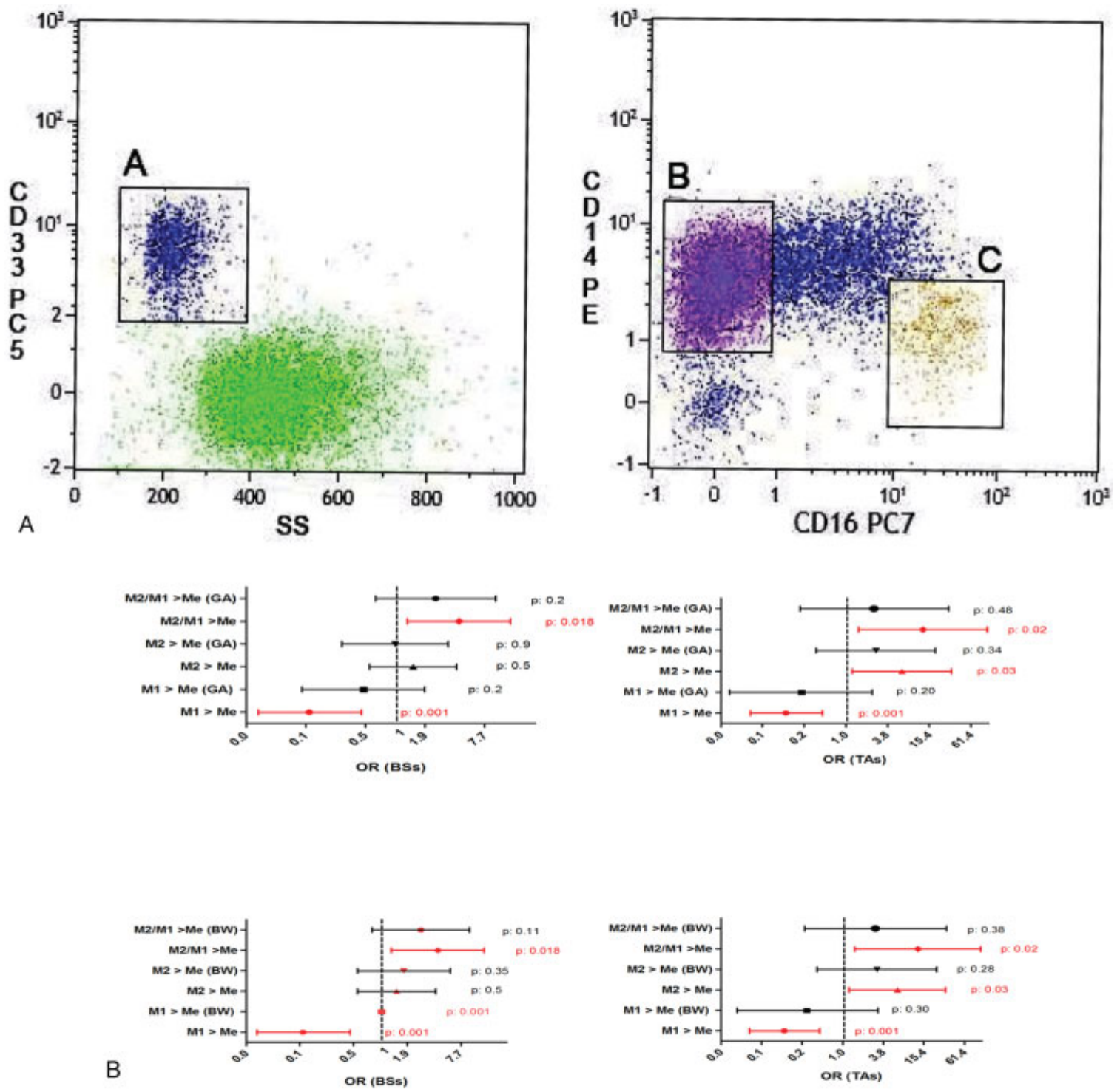

Fig. 1 (A) Flow cytometer. Isolation of macrophages by CD33, followed by using CD14 and CD16 to identify CAM ed AAM. (Square A: macrophages CD33+/SS low; square B: CAM, M2, CD14++/CD16-; and square C: AAM, M1, CD14+/CD16+). (B) After turning macrophages pools into categorical variables through "median split" categorization the association with development of BPD was calculated. By the binary logistic regression, a positive relation (OR $>1$ ) was found among $M 2$ and $M 2 / M 1$ with BPD, negative $(O R<1)$ between $M 1$ and $B P D$, in the BSs), and TAs. Whether binary logistic regression was corrected by GA and BW, the relation with BPD lacks in significance. Odds and confidence intervals are shown; red is significant. Square A: blue, macrophages CD33+/side scatter low; green are granulocytes; square B: violet, CAM, M2, CD14+/CD16-; square C: yellow, AAM, M1, CD14+CD16+. AAM, alternatively activated macrophages; BPD, bronchopulmonary dysplasia; BSs, blood samples; BW, birth weight; CAM, classically activated macrophages; GA gestational age; OR, odds ratio; SSlow, side scatter low; TAs, tracheal aspirates.

Internal quality control (IQC), the so-called Immunotrol, performed daily, consisted of a liquid preparation of human stabilized red and white blood cells that was processed as a routine sample with the reagents in use. If the IQC was within the acceptable range, sample acquisition and analysis were performed. The UK Neqas External Quality Assessment "Leucocyte Immunophenotyping” external QC was performed.

\section{Ethical Approval}

Ethical approval for the study was obtained from the local ethics committee (AOP 2724P/Nov 2012), and informed consent was obtained from parents.

\section{Statistical Analysis}

Mean and standard deviation (SD) values are reported for continuous variables, whereas for categorical variables, percentage values are presented. Since the normal distribution of macrophages sub-types (M1 and M2) was confirmed by the Kolmogorov normality test, their association with other variables has been analyzed by mean of $t$-test, linear regression, and variance analysis (Dell Statistics 13 and Graph Pad 6 Software). Odds ratio for BPD have been calculated, after categorization of macrophages levels through "median split" technique, by mean of a model of binary logistic regression (IBM SPSS Software). Significance level was set at $p<0.05$. 


\section{Results}

A total of 108 preterm newborns ( 49 male and 59 female) were included in the study, with a mean GA of $29^{+1 / 7}$ weeks and a BW of $1274 \mathrm{~g}$. Among them, 49\% were born before 28 gestational weeks, $87 \%$ after CS, $22 \%$ after chorioamnionitis, $25 \%$ were twins, and $77 \%$ had received antenatal steroids. At the time of sampling, almost all subjects had RDS (mid-degree) with a mean $\mathrm{FiO}_{2}$ of 0.31 .

One hundred patients were followed up, mean $\mathrm{MV}$ and $\mathrm{O}_{2}$ therapy duration were 9.1 and 17.8 days, respectively, and BPD was diagnosed in 22 of them (-Table 1). TAs and BSs were adequate for flow cytometry analysis in 96/108 and 97/108 patients, respectively.

\section{Macrophage Profile and Prenatal Factors}

Unaffected newborns showed a higher level of M1 cells versus maternal chorioamnionitis newborns in BSs $(p=0.013$, - Fig. 2A 1$)$ and TAs $(p=0.007,-$ Fig. 2A 4$)$ and lower levels of M2 ( $p=0.02$, -Fig. 2A5) and M2/M1 $(p=0.004$, -Fig. 2A6). CS, twins pregnancy, and antenatal steroid administration did not show differences except for a higher level of M2 and M2/M1 in the BSs of patient born from CS $(p<0.05)$ and a higher level of M2 in TAs $(p<0.01)$ after antenatal steroids ( - Table 2 and 3, - Fig. 2 ).

Table 1 Clinical data of the studied newborns

\begin{tabular}{|l|l|}
\hline Clinical data & Mean (SD); $\boldsymbol{n}(\%)$ \\
\hline Overall & 108 \\
\hline Female & $59(54)$ \\
\hline GA & $29 \mathrm{wk}^{+1 / 7} \mathrm{~d}\left(3 \mathrm{wk}^{+1} \mathrm{~d}\right)$ \\
$<28 \mathrm{wk}$ & $53(49)$ \\
$>28 \mathrm{wk}$ & $55(51)$ \\
\hline BW (g) & $1274.1(648.6)$ \\
\hline CS & $94(87)$ \\
\hline Twins & $27(25)$ \\
\hline Chorioamnionitis & $19 / 85(22.3)$ \\
\hline Antenatal steroids & $77(71.3)$ \\
\hline RDS & $105(97.2)$ \\
Mid degree & $2.1(0.8)$ \\
\hline FiO ${ }_{2}$ (\%) & $0.31(16.7)$ \\
\hline Surfactant & $85(78.7)$ \\
\hline Surfactant doses/newborn & $1.6(0,8)$ \\
\hline Followed-up & $100(92.5)$ \\
\hline MV (d) & $9.1(11.8)$ \\
\hline O $_{2}$ therapy (d) & $17.8(25.6)$ \\
\hline O 2 therapy plus ventilation & $42(42)$ \\
\hline at 28 d & $22(22)$ \\
\hline BPD & \\
\hline
\end{tabular}

Abbreviations: BW, birth weight; BPD, bronchopulmonary dysplasia; CS, cesarean section; GA gestational age; MV, mechanical ventilation; RDS, respiratory distress syndrome; SD, standard deviation.

\section{Macrophage profile and biological features:}

BSs and TAs show a direct correlation between levels of M1 with GA (-Fig. 3) (BSs: $R^{2}=0.40, p<0.001$, -Fig. 3A1; TAs: $R^{2}=0.40, p<0.001,-$ Fig. 3A4). Similarly M1 correlate with BW (BSs: $R^{2}=0.42, p<0.001$, - Fig. 3B1; TAs: $R^{2}=0.42$, $p<0.001$, - Fig. 3B4). Levels of M2 are instead inversely correlated with GA (BSs: $R^{2}=0.06, \mathrm{p}<0.014$, - Fig. 3A2; TAs: $R^{2}=0.23, p<0.001$, - Fig. 3A5) and with BW (BSs: $R^{2}=0.06, \quad p=0.016, \quad$-Fig. $\quad 3 \mathrm{~B} 2 ; \quad$ TAs: $R^{2}=0.26$, $p<0.001$, - Fig. 3B5). The same applies to M2/M1 with GA (BSs: $R^{2}=0.14, p<0.001$, -Fig. 3A3; TAs: $R^{2}=0.24$, $p<0.001$, -Fig. 3A6) and with BW (BSs: $R^{2}=0.20$, $p=0.003$, -Fig. 3B3; TAs: $R^{2}=0.24, p<0.001$, -Fig. 3B6).

\section{Macrophage profile and respiratory distress syndrome:}

Variance analysis did not show any significant difference between macrophage distribution and RDS grades. Similarly, linear regression failed to reveal any correlation between macrophage subtypes and $\mathrm{FiO}_{2}$ at the time of sampling.

\section{Macrophage profile and chronic lung disease}

Linear regression analysis shows an inverse correlation between levels of M1 and MV time (-Fig. 2 and 4, - Table 4) (BSs: $R^{2}=0.20, \quad p<0.001, \quad$-Fig. $\quad 4 \mathrm{~A} 1 ; \quad$ TAs: $R^{2}=0.23$, $p<0.001$, - Fig. 4A4) as well as $\mathrm{O}_{2}$ therapy duration (BSs: $R^{2}=0.20, \quad p<0.001, \quad$-Fig. $\quad 4 \mathrm{~B} 1 ; \quad$ TAs: $\quad R^{2}=0.10$, $p<0.001$, - Fig. 4B4). Levels of M2 are directly correlated with MV time (BSs: $R^{2}=0.05, p=0.04$, - Fig. 4A2; TAs: $R^{2}=0.20, p<0.001$, - Fig. 4A5) and $\mathrm{O}_{2}$ therapy duration (BSs: $R^{2}=0.07, \quad p=0.01$, -Fig. 4B2; TAs: $R^{2}=0.12$, $p<0.009$, - Fig. 4B5). Similarly M2/M1 increase with duration of MV (BSs: $R^{2}=0.10, p=0.002$, - Fig. 4A3; TAs: $R^{2}=0.23$, $p<0.001$, -Fig. 4A6) and of $\mathrm{O}_{2}$ duration (BSs: $R^{2}=0.16$, $p<0.001$, -Fig. 4B3; TAs: $R^{2}=0.06, p=0.06$, -Fig. 4B6). Consistently, samples from patients who subsequently developed BDP show at birth lower levels of M1 (BSs: $p=0.000$, -Fig. 2B1; TAs: $p=0.000$, -Fig. 2B4), increased levels of M2 (BSs: NS, - Fig. 2B2; TAs $p=0.003$, - Fig. 2B5) and of M2/M1 (BSs: $p=0.005$, -Fig. 2B3; TAs: $p=0.005$, - Fig. 2B6). After categorization of variables referred to levels of macrophages subtypes through "median split", the association with presence or absence of BPD was analyzed by means of binary logistic regression. When M2/M1 is over the median value, the odds for BPD are from 4.3 (BSs, $p=0.016$ ) to 15.5 (TAs, $p=0.02$ ) times higher. When corrected for GA and BW, the odds for BPD are not statistically significant (-Fig. 1B).

\section{Discussion}

Despite notable advances in management of preterm birth, BPD still represents a major complication with an important short- and long-term burden on quality of life of affected newborn infants. A variety of prenatal and postnatal injuries ( $\mathrm{MV}, \mathrm{O}_{2}$ toxicity, infections, patent ductus arteriosus, fluid overload, chorioamnionitis, and nutritional deficits) impaired the lung, which is immature and not able to react 

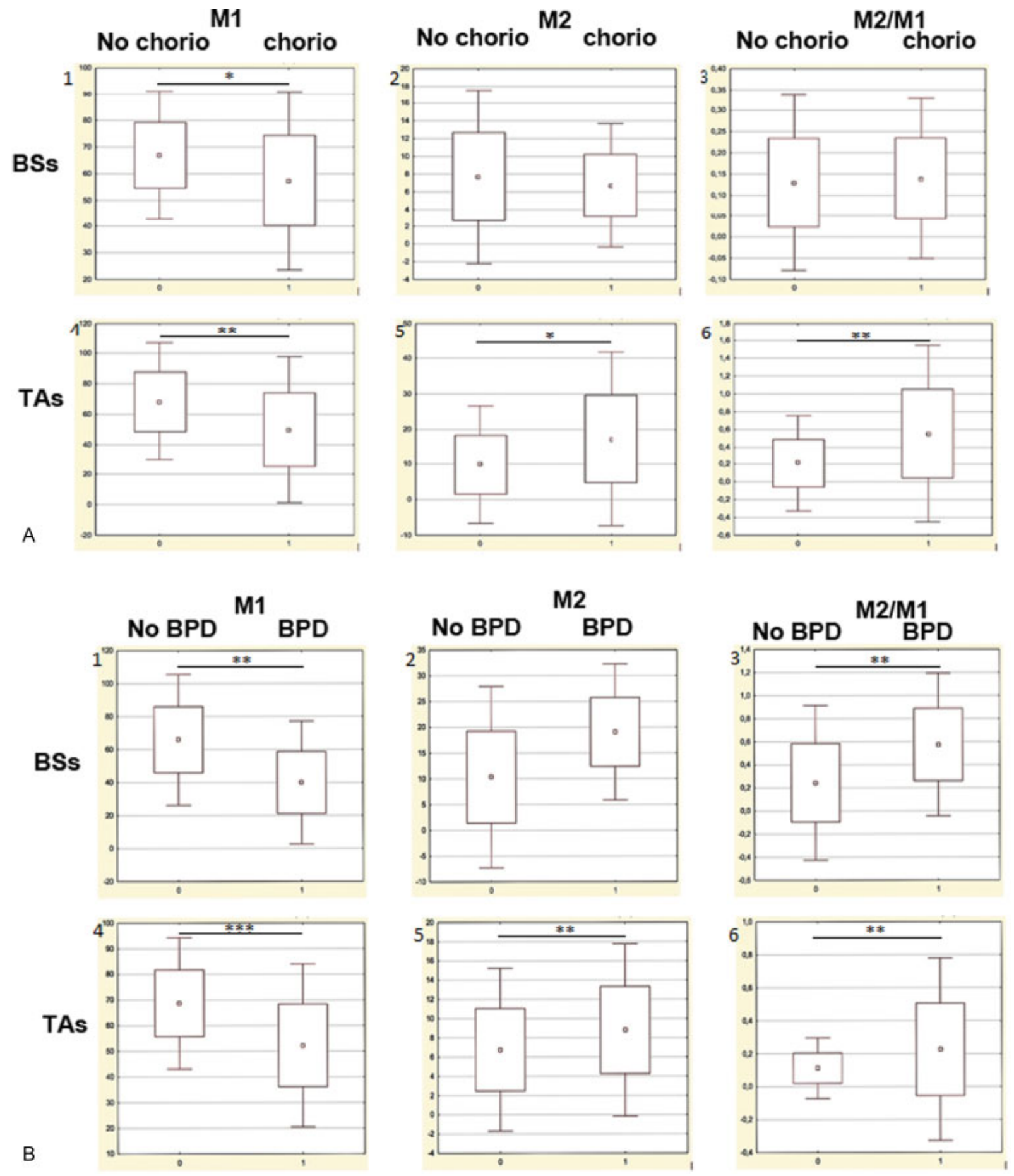

Fig. 2 (A) Chorioamnionitis and macrophages from blood samples (BSs) and tracheal aspirates, (TAs). Box and whiskers plots show the level of $\mathrm{M} 1, \mathrm{M} 2$, and M2/M1 in the BSs (1-3 squares) and TAs (4-6 squares). Horizontal axes show 0: no chorioamnionitis and 1: chorioamnionitis. M1 percentages decrease in the BSs and TAs from neonates born under chorioamnionitis, when compared to the unaffected. No changes were found for $\mathrm{M} 2$ or $\mathrm{M} 2 / \mathrm{M} 1$ in the BSs, against in the TAs $\mathrm{M} 2$, and M2/M1 rose in newborns under chorioamnionitis. (B) Bronchopulmonary dysplasia (BPD), and macrophages samples from BSs and TAs. Box and whiskers plots show the level of M1, M2, and M2/M1 in the BSs (1-3 squares) and TAs (4-6 squares). Horizontal axes show 0: no BPD and 1: BPD. M2/M1 increase in the BSs and TAs from neonates who develop BPD, when compared to the unaffected. M1 decrease in newborns that develop BPD, either in the BSs or TAs, against M2 increase in TAs of the BPD patients. BPD, bronchopulmonary dysplasia; BSs, blood samples; TAs, tracheal aspirates.

adequately to the pathological noxae. Many of the affected patients are extremely premature who are exposed to prolonged ventilation for lung RDS, but others will initially have few signs of respiratory disease.
While the goal in BPD therapy would be to promote a full structural and functional recovery guiding a correct lung development, current therapeutic approaches mainly treat symptoms and are of limited benefit. 
Table 2 Chorioamnionitis and macrophages from BSs and TAs

\begin{tabular}{|c|c|c|c|c|c|c|}
\hline Mean (SD) & $\begin{array}{l}\text { Chorioamnionitis } \\
(n=16) \text { BSs }\end{array}$ & $\begin{array}{l}\text { No chorioamnionitis } \\
(n=58) \mathrm{BSs}\end{array}$ & $p$ & $\begin{array}{l}\text { Chorioamnionitis } \\
(n=13) \text { TAs }\end{array}$ & $\begin{array}{l}\text { No chorioamnionitis } \\
(n=39) \text { TAs }\end{array}$ & $p$ \\
\hline M1 & $57.3(17.2)$ & $66.9(12.4)$ & 0.013 & $49.5(24.6)$ & $68.1(19.7)$ & 0.007 \\
\hline M2 & $6.67(3.6)$ & $7.67(5.00)$ & NS & $17.2(12.5)$ & $9.90(8.49)$ & 0.02 \\
\hline M2/M1 & $0.14(0.09)$ & $0.13(0.10)$ & NS & $0.54(0.50)$ & $0.21(0.27)$ & 0.004 \\
\hline
\end{tabular}

Abbreviations: BSs, blood samples; SD, standard deviation; TAs, tracheal aspirates.

Note: M1 percentages decrease in the BSs and TAs from neonates born under chorioamnionitis, when compared to the unaffected. No changes were found for $\mathrm{M} 2$ or M2/M1 in the BSs, against in the TAs M2, and M2/M1 rose in newborns under chorioamnionitis.

Table 3 AS and macrophages from BSs and TAs: M2 rose under AS treatment

\begin{tabular}{|c|c|c|c|c|c|c|}
\hline Mean (SD) & $\begin{array}{l}\text { AS } \\
(n=20) \\
\text { BSs }\end{array}$ & $\begin{array}{l}\text { No AS } \\
(n=70) \\
\text { BSs }\end{array}$ & $p$ & $\begin{array}{l}\text { AS } \\
(n=42) \\
\text { TAs }\end{array}$ & $\begin{array}{l}\text { No AS } \\
(n=19) \\
\text { TAs }\end{array}$ & $p$ \\
\hline M1 & $63.4(19.1)$ & $64.3(13.4)$ & NS & $60.8(21.8)$ & $61.1(23.5)$ & NS \\
\hline M2 & $7.5(5.6)$ & $7.3(4.1)$ & NS & $14.8(6.7)$ & $6,3(5.8)$ & 0.01 \\
\hline $\mathrm{M} 2 / \mathrm{M} 1$ & $0.17(0.30)$ & $0.13(0.30)$ & NS & $0.31(0.34)$ & $0.29(0.41)$ & NS \\
\hline
\end{tabular}

Abbreviations: AS, antenatal steroids; BSs, blood samples; SD, standard deviation; TAs, tracheal aspirates.

The innate immune system, particularly the monocytemacrophage system, is known to play a crucial role not only in first line defense against pathogens, but also in tissue remodeling and repair processes. Cells, such as M1, can react to the stimulus with a non-specific response aimed to remove pathogens, dead cells, and debris by phagocytosis. Only after this first phase, cells with reparative and immunosuppressive effect can take part in the process of tissue remodeling and restoring; among them are the M2. We hypothesized that these self-defense and reparative mechanisms based on macrophages activation could be involved in BPD development, as suggested by a previous study on animal model. ${ }^{2}$

Airway macrophages mostly differentiate after birth, but they can be detected also in fetal lung where they differentiate under the influence of the local microenvironment (cytokines, surfactant, alveolar cells, and bronchial epithelium). ${ }^{16}$

Our analysis showed that macrophage activation pattern at birth changed with GA: in our population, a low GA and a low BW correlate with higher levels of M2 and lower levels of M1 cells, both in BSs and TAs. We can speculate that in the preterm newborn, the macrophage system is immature, not capable to push undifferentiated macrophages toward a classical activation pathway. Our analysis would be consistent with previous findings, which show a deficit of the immune system, from a qualitative rather than a quantitative point of view.

Available data so far show a defective immune response type Th1 in favor of a Th2 response in newborn and in particular in those who were born prematurely. ${ }^{17,18}$ In addition, also cytokines profile is different, characterized by a deficit of pro-inflammatory IL- 1 or Il- 6 and TNF- $\alpha$ production, ${ }^{19,20}$ with a prevalence of anti-inflammatory IL-10 and TGF- $\beta .^{21,22}$ Our results are in line with the hypothesis that preterm newborns are defective in macrophage function and activation, related to impaired cytokines production. ${ }^{19}$
Furthermore, the association we found between more preterm delivery and higher level of M2 agrees with recent studies that compared term and preterm macrophages activation status. ${ }^{13}$ This condition could be influenced by the immune tolerance state present at the maternal-fetal interface level during pregnancy. $^{23}$ Maternal macrophages activation has been widely studied both in physiological and pathological pregnancy since they seem to play a crucial role. After an early inflammatory phase in which macrophage undergo an M1 polarization to ensure the trophoblastic implantation, a transition to the predominant M2 profile characterizes the second and third trimesters. It has been suggested that this macrophage state is able to set up an immunosuppressive environment to prevent the fetal reject. ${ }^{24,25}$ Finally, the last phase of physiological gestation sees a rise in inflammatory cytokines and M1 cells during labor. This late gestation shift of the macrophage activation status could explain the low M1 level in preterm newborn, as a result of the uterine anti-inflammatory response at that stage of pregnancy.

The analysis of association between macrophage subsets and chorioamnionitis has shown a higher M2 level in the group of patients born after chorioamnionitis. This result may sound unexpected since during an infectious disease an inflammatory response with activation of M1 cells would be expected. Nonetheless, available data in the literature have not shown any M1 polarization during chorioamnionitis at the maternal-fetal interface. ${ }^{24,26}$

In conclusion, we can assume that the evolutionary advantage of an attenuated inflammatory response in utero, necessary for an adequate fetal growth, could reflect on the immune system state of the preterm newborn.

Analyzing the relation between macrophages and the severity of acute RDS, no association has been found with both radiological picture and $\mathrm{O}_{2}$ levels. This is possibly due to 

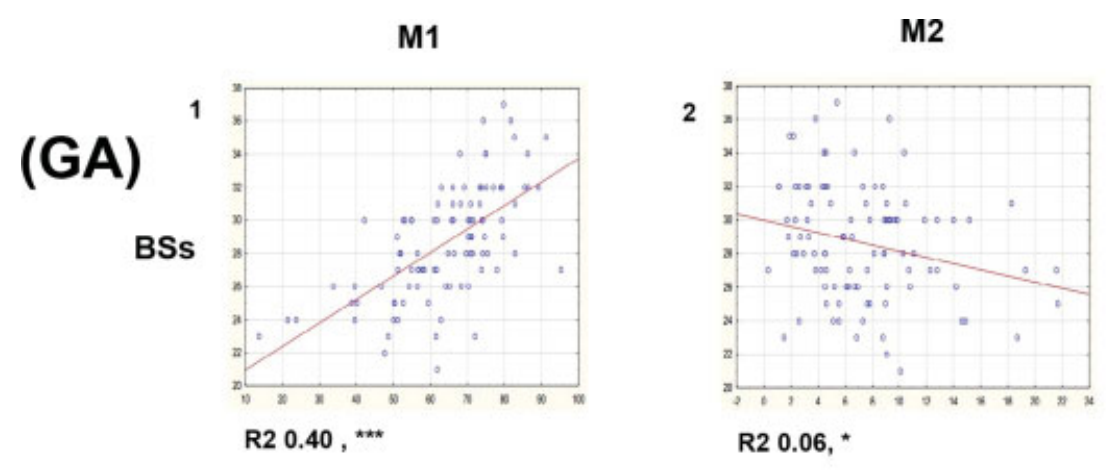

R2 0.06, *

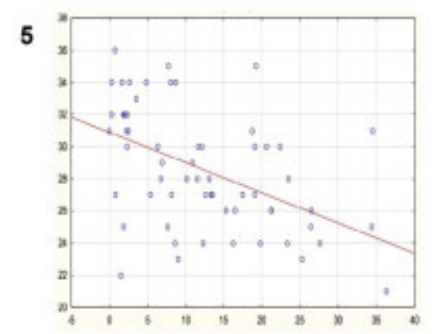

R2 0.23, $\cdots$

A

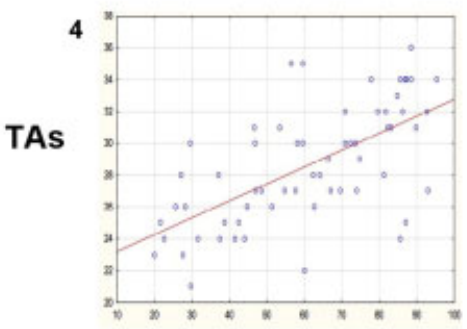

R2 0.40, ***

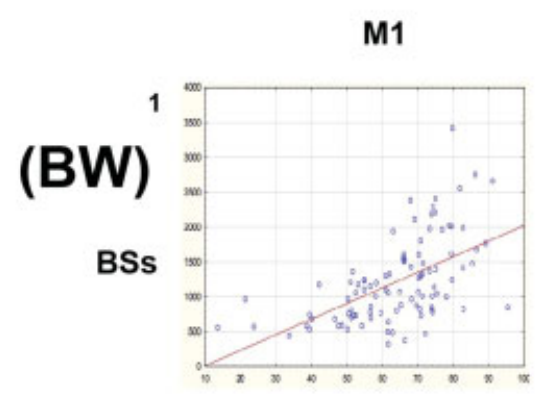

R2 0.42, ***

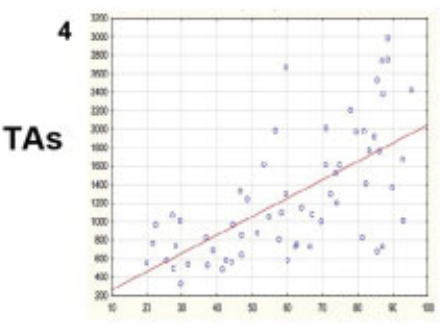

B

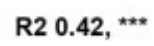

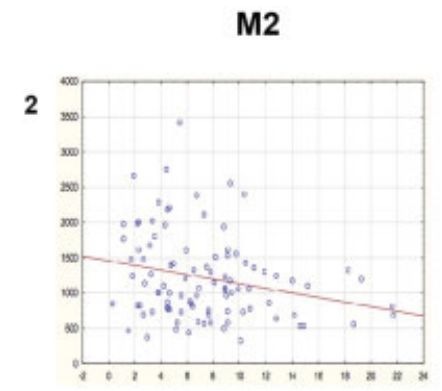

R2 0.06, *

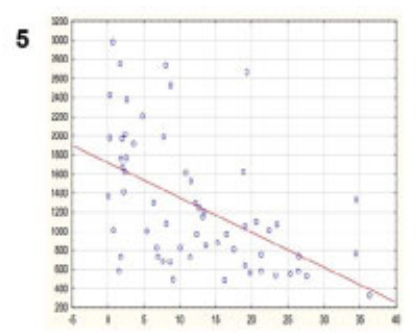

R2 0.26, ***
M2/M1

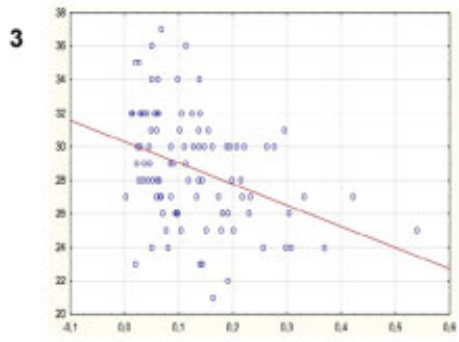

R2 $0.14, *$

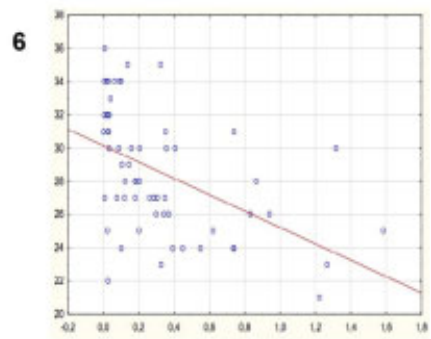

R2 0.24, ***

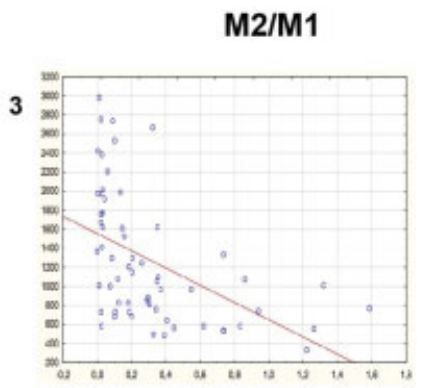

R2 0.20, **

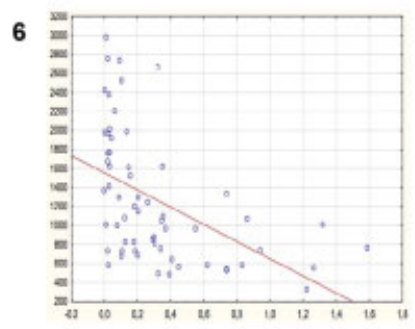

R2 0.24, ***

Fig. 3 (A) Linear regressions between GA with macrophages percentage in the BSs (1-3 squares) and TAs (4-6 squares). (B) Linear regressions between BW with macrophages percentage in the BSs (1-3 squares) and TAs (4-6 squares). The best relation is found between M1 with GA and with BW in the BSs and TAs. Against the $\mathrm{M} 2$ and M2/M1 (from BSs and TAs) are decreasing with GA and BW. BW, birth weight; GA gestational age; TAs, tracheal aspirates.

Table 4 BPD and macrophages from BSs and TAs

\begin{tabular}{|l|l|l|l|l|l|l|}
\hline Mean (SD) & $\begin{array}{l}\text { BPD } \\
(\boldsymbol{n}=\mathbf{2 0}) \text { BSs }\end{array}$ & $\begin{array}{l}\text { No BPD } \\
(\boldsymbol{n}=\mathbf{6 7}) \text { BSs }\end{array}$ & $\boldsymbol{p}$ & $\begin{array}{l}\text { BPD } \\
(\boldsymbol{n}=\mathbf{1 1}) \text { TAs }\end{array}$ & $\begin{array}{l}\text { No BPD } \\
(\boldsymbol{n}=\text { 45) TAs }\end{array}$ & $\boldsymbol{p}$ \\
\hline M1 & $52.2(16.2)$ & $68.7(13.0)$ & 0.000 & $39.9(19.0)$ & $65.9(20.2)$ & 0.000 \\
\hline M2 & $8.81(4.60)$ & $6.7(4.30)$ & 0.07 & $19.1(6.70)$ & $10.3(9.00)$ & 0.003 \\
\hline M2/M1 & $0.22(0.28)$ & $0.11(0.09)$ & 0.005 & $0.57(0.31)$ & $0.24(0.34)$ & 0.005 \\
\hline
\end{tabular}

Abbreviations: BPD, bronchopulmonary dysplasia; BSs, blood samples; SD, standard deviation; TAs, tracheal aspirates.

Note: M2/M1 increase in the BSs and TAs from neonates who develop BPD, when compared to the unaffected. M1 decrease in newborns that develop $B P D$, either in the BSs and TAs, against M2 decrease in BPD patients. 
M1

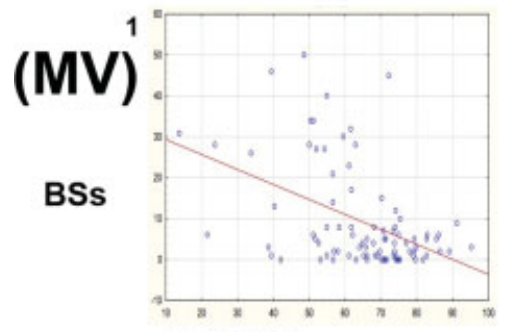

R2 0.20 , ***

TAs

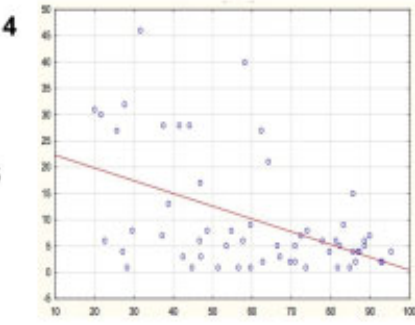

A

R2 0.23, ****

M1

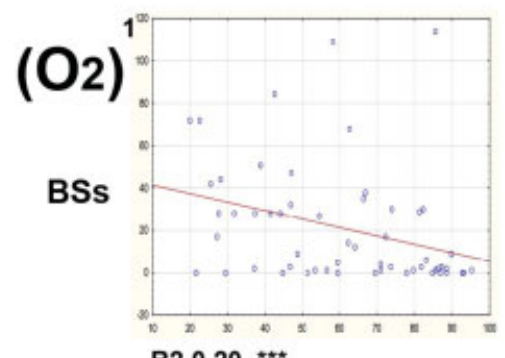

R2 0.20, ***

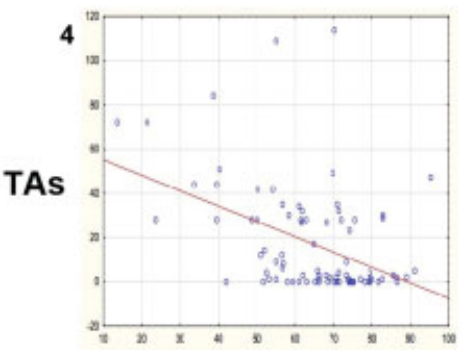

B

R2 0.10, ***
M2

2

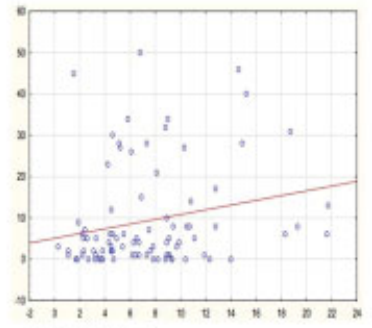

R2 0.05, *

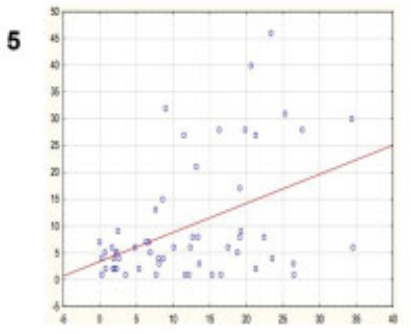

R2 0.20 , ***

M2

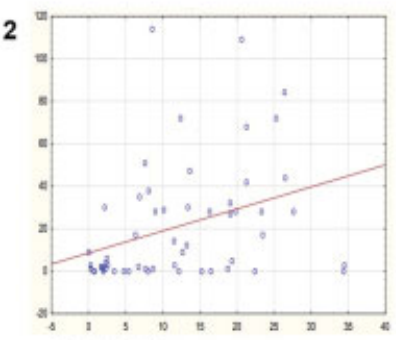

R2 0.07, *

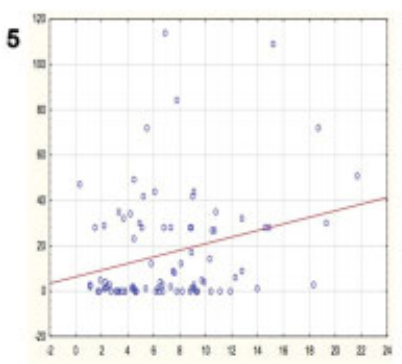

R2 0.12, *
M2/M1

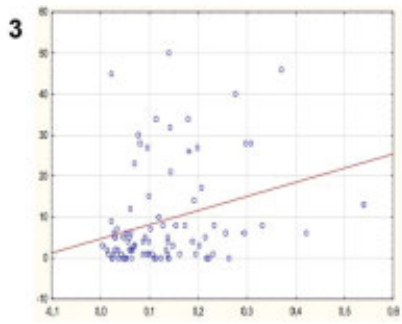

R2 $0.10, \cdots$

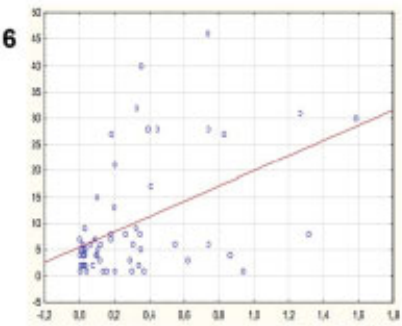

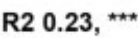

M2/M1

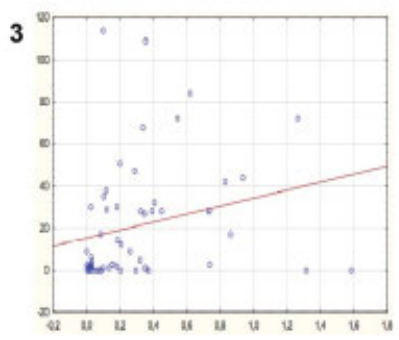

R2 0.16, ***

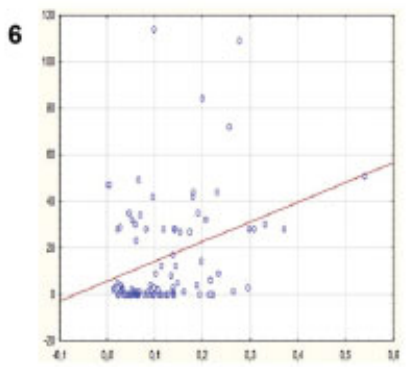

R2 0.06 , NS

Fig. 4 (A) Linear regressions between the duration of the MV and the macrophages percentage in the BSs (1-3 squares) and TAs (4-6 squares). (B) Linear regressions between the duration of $\mathrm{O}_{2}$ therapy and the macrophages percentage in the BSs (1-3 squares) and TAs (4- 6 squares). The best relation is found between $\mathrm{M} 2$ and $\mathrm{M} 2 / \mathrm{M} 1$ with $\mathrm{MV}$ and $\mathrm{O}_{2}$ therapy in the BSs and TAs. Against the $\mathrm{M} 1$ is inversely related (from BSs and TAs) with $M V$ and $\mathrm{O}_{2}$ duration. BSs, blood samples; MV, mechanical ventilation; TAs, tracheal aspirates.

the presence of many contributing factors that determine RDS severity, e.g., surfactant administration and concomitant pneumonia. Conversely, predictors of BPD, such as MV and $\mathrm{O}_{2}$ duration, were associated to both a higher level of M2 and that of M2/M1. Our study shows that babies who do not develop BPD have lower M2 levels and M2/M1 both in BSs and TAs in the first days of life, with higher M1 level. This predominant classical activation of the macrophage population could represent an appropriate early reaction to the injury with inflammatory and first-line cells recruitment. On the other side, the lack of this immediate response could expose the lungs to a more severe damage. Moreover, it affects the activation of secondary phase cells, reducing the possibility of tissue repair. It is important to remember that 
our study analyses the early activation of macrophages, not the inflammatory state at the time of BPD assessment. BPD itself has been described for a long time as an inflammatory mediated disease, but, especially in the so called "new BPD",27,28 the primum movens of the disease is far from being a simple inflammatory reaction and is more related to a simplification of the lung structure due to the vascular and alveolar immaturity. An adequate inflammatory reaction should not be simply considered as a cause of tissue damage; on the contrary, it represents the first-line response to the injury without which no tissue repair could be subsequently achieved. Neonates who cannot promote these mechanisms of self-defense and have lower M1 levels during the first days of life could be more susceptible to BPD. Consequently, the macrophage activation status could predict the BPD development. We have observed that when the M2/M1 exceeds the median value, BPD odds increases 4 to 15 times, respectively, for TAs and BSs. The statistical significance of this association failed when corrected for GA and BW. Consequently, we also assume cautiously the macrophages' polarization as predictor variable toward BPD development.

The main limitations of the present study are related to the analysis of macrophages level at a single time point and the impossibility to monitor the macrophage system activation during the development of BPD.

To better analyze the role of M1 and M2 in BPD, further studies should be designed to detect the level of multiple cytokines involved in macrophages activation and differentiation. The level we found in macrophages pools may show a time-related polarization or gene expression, which interplay with more complicated pathways in the alveolar and vascular lung architecture, i.e., VEGF, ${ }^{29}$ $\mathrm{NOTCH},{ }^{30}$ and other crucial signaling for vascular and alveolar morphogenesis. ${ }^{31}$

In conclusion, our study has analyzed the monocytemacrophage system in a group of premature babies showing a deficit of classical activation pathway in the infants born at the earliest gestational age. In addition, babies who developed BPD presented, at birth, a lower level of classical activated macrophages and higher levels of alternative activated macrophages. We speculate that in preterm infants the innate immune system is immature and unable to push undifferentiated macrophages toward the classical activation pathway, and it is still affected by the immune tolerance status of the maternal-fetal interface. ${ }^{32}$ In this context, early classical macrophage activation could be seen as not only a reaction to lung injury, but most importantly, as a crucial step to start an appropriate defense-repair response to protect the lungs from post-natal injuries, preventing the BPD. A paradoxical increase in M1 polarization seems to correlate with a normal lung growth. However, we know that M1 pool makes enhanced oxidant production, carrying out the activation of fatty acids synthesis, which directly contributes to the inflammatory response. $^{33}$ In addition, it is well known that this M1 activation, associated with production of reactive oxygen species and inflammatory cytokines, can substantially impair vascular function. Indeed, impaired microcirculatory function in newborns with respiratory distress syndrome has been directly documented. ${ }^{9}$ An increase in M2 was found under antenatal maternal steroids. This data disagree with the assumption that several maturational steps, more than the influence from perinatal factors, are challenging in the immune defense in very preterm newborns during the neonatal period. ${ }^{34}$

Since M1 mechanism is already well known as to be proinflammatory, we wonder whether this early mechanism results to be protective in the late lung development pathways. Furthermore, a "time-related inflammation" in lung macrophages pools may exert several responses in the function of the preterm immune system, and more interestingly, it seems to participate differently in lung development and differentiation of alveolar epithelial cells. However, the macrophages are still so far to show some diagnostic value and to retain some therapeutic applications. Further studies are needed to confirm this hypothesis and to assess the role of cytokines involved in macrophages activation.

\section{Note}

The study is a clinical research, conducted with financial and ethic supports of the University Padova Hospital (AOP 2724P/Nov 2012).

\section{Conflict of Interest}

None.

\section{Acknowledgments}

The authors are very grateful to Prof. Lino Chiandetti for the useful advice in performing the overall study design. They thank Drs. M.C. Sanzari, V. Vanzo, and E. Franchetti for technical support. In addition, the authors are indebted to the nurses of the NICU for the invaluable help.

\section{References}

1 Stoll BJ, Hansen NI, Bell EF, et al; Eunice Kennedy Shriver National Institute of Child Health and Human Development Neonatal Research Network. Neonatal outcomes of extremely preterm infants from the NICHD Neonatal Research Network. Pediatrics 2010;126(03):443-456

2 Balasubramaniam V, Ryan SL, Seedorf GJ, et al. Bone marrowderived angiogenic cells restore lung alveolar and vascular structure after neonatal hyperoxia in infant mice. Am J Physiol Lung Cell Mol Physiol 2010;298(03):L315-L323

3 Geissmann F, Jung S, Littman DR. Blood monocytes consist of two principal subsets with distinct migratory properties. Immunity 2003;19(01):71-82

4 Gordon S, Taylor PR. Monocyte and macrophage heterogeneity. Nat Rev Immunol 2005;5(12):953-964

5 Dale DC, Boxer L, Liles WC. The phagocytes: neutrophils and monocytes. Blood 2008;112(04):935-945

6 Chambers SE, O'Neill CL, O'Doherty TM, Medina RJ, Stitt AW. The role of immune-related myeloid cells in angiogenesis. Immunobiology 2013;218(11):1370-1375

7 Varin A, Gordon S. Alternative activation of macrophages: immune function and cellular biology. Immunobiology 2009;214 (07):630-641

8 Novak ML, Koh TJ. Macrophage phenotypes during tissue repair. J Leukoc Biol 2013;93(06):875-881 
9 Troiani S, Cardona A, Milioni M, et al. Evidence of impaired microvascular dilatation in preterms with acute respiratory distress syndrome. Int J Cardiol 2017;241:83-86

10 Redline RW, Faye-Petersen O, Heller D, Qureshi F, Savell V, Vogler C; Society for Pediatric Pathology, Perinatal Section, Amniotic Fluid Infection Nosology Committee. Amniotic infection syndrome: nosology and reproducibility of placental reaction patterns. Pediatr Dev Pathol 2003;6(05):435-448

11 Agrawal V, David RJ, Harris VJ. Classification of acute respiratory disorders of all newborns in a tertiary care center. J Natl Med Assoc 2003;95(07):585-595

12 Ehrenkranz RA, Walsh MC, Vohr BR, et al; National Institutes of Child Health and Human Development Neonatal Research Network. Validation of the National Institutes of Health consensus definition of bronchopulmonary dysplasia. Pediatrics 2005;116 (06):1353-1360

13 Prince LR, Maxwell NC, Gill SK, et al. Macrophage phenotype is associated with disease severity in preterm infants with chronic lung disease. PLoS One 2014;9(08):e103059

14 Gratama JW, Kraan J, Keeney M, Granger V, Barnett D. Reduction of variation in T-cell subset enumeration among 55 laboratories using single-platform, three or four-color flow cytometry based on CD45 and SSC-based gating of lymphocytes. Cytometry 2002; 50(02):92-101

15 Novak ML, Weinheimer-Haus EM, Koh TJ. Macrophage activation and skeletal muscle healing following traumatic injury. J Pathol 2014;232(03):344-355

16 Iliodromiti Z, Anastasiadis A, Varras M, et al. Monocyte function in the fetus and the preterm neonate: immaturity combined with functional impairment. Mediators Inflamm 2013;2013:753752

17 Fleer A, Krediet TG. Innate immunity: toll-like receptors and some more. A brief history, basic organization and relevance for the human newborn. Neonatology 2007;92(03):145-157

18 Maródi L. Innate cellular immune responses in newborns. Clin Immunol 2006;118(2-3):137-144

19 Currie AJ, Curtis S, Strunk T, et al. Preterm infants have deficient monocyte and lymphocyte cytokine responses to group B streptococcus. Infect Immun 2011;79(04):1588-1596

20 Yachie A, Takano N, Ohta K, et al. Defective production of interleukin-6 in very small premature infants in response to bacterial pathogens. Infect Immun 1992;60(03):749-753
21 Short EJ, Kirchner HL, Asaad GR, et al. Developmental sequelae in preterm infants having a diagnosis of bronchopulmonary dysplasia: analysis using a severity-based classification system. Arch Pediatr Adolesc Med 2007;161(11):1082-1087

22 Strunk T, Temming P, Gembruch U, Reiss I, Bucsky P, Schultz C. Differential maturation of the innate immune response in human fetuses. Pediatr Res 2004;56(02):219-226

23 Tang MX, Hu XH, Liu ZZ, Kwak-Kim J, Liao AH. What are the roles of macrophages and monocytes in human pregnancy? J Reprod Immunol 2015;112:73-80

24 Brown MB, von Chamier M, Allam AB, Reyes L. M1/M2 macrophage polarity in normal and complicated pregnancy. Front Immunol 2014;5:606

25 Cappelletti M, Della Bella S, Ferrazzi E, Mavilio D, Divanovic S. Inflammation and preterm birth. J Leukoc Biol 2016;99(01): $67-78$

26 Gomez-Lopez N, Romero R, Arenas-Hernandez M, et al. In vivo Tcell activation by a monoclonal $\alpha C D 3 \varepsilon$ antibody induces preterm labor and birth. Am J Reprod Immunol 2016;76(05):386-390

27 Baraldi E, Filippone M. Chronic lung disease after premature birth. N Engl J Med 2007;357(19):1946-1955

28 Coalson JJ. Pathology of bronchopulmonary dysplasia. Semin Perinatol 2006;30(04):179-184

29 Porzionato A, Zaramella P, Macchi V, et al. Fluoxetine may worsen hyperoxia-induced lung damage in neonatal rats. Histol Histopathol 2012;27(12):1599-1610

30 Singla DK, Wang J, Singla R. Primary human monocytes differentiate into M2 macrophages and involve Notch-1 pathway. Can J Physiol Pharmacol 2017;95(03):288-294

31 Thébaud B, Abman SH. Bronchopulmonary dysplasia: where have all the vessels gone? Roles of angiogenic growth factors in chronic lung disease. Am J Respir Crit Care Med 2007;175(10):978-985

32 Zhang YH, He M, Wang Y, Liao AH. Modulators of the balance between M1 and M2 Macrophages during Pregnancy. Front Immunol 2017;8:120

33 Ménégaut L, Thomas C, Lagrost L, Masson D. Fatty acid metabolism in macrophages: a target in cardio-metabolic diseases. Curr Opin Lipidol 2017;28(01):19-26

34 Marchant EA, Kan B, Sharma AA, et al. Attenuated innate immune defenses in very premature neonates during the neonatal period. Pediatr Res 2015;78(05):492-497 